\title{
Predictive maintenance of maritime systems: models and challenges
}

\author{
T. Tinga, W.W. Tiddens \& F. Amoiralis \\ Netherlands Defence Academy, Den Helder, Netherlands \&
}

University of Twente, Enschede, Netherlands

M. Politis

Netherlands Defence Academy, Den Helder, Netherlands \&

Eindhoven University of Technology, Eindhoven, Netherlands

\begin{abstract}
To reduce maintenance and logistic costs and increase the asset availability, a predictive maintenance concept for maritime systems is developed. In the present paper, the physics-of-failure based prognostic methods will be introduced, but also other issues related to the development and application of these models will be discussed. Typically, the following challenges are encountered in such a development trajectory: (i) critical part selection, (ii) predictive modelling (data-driven or physics based), (iii) monitoring / data collection, (iv) model validation and (v) making the business case. These challenges will be discussed using two case studies: the cylinder liners of a diesel engine and printed circuit boards (PCB) in a radar system.
\end{abstract}

\section{INTRODUCTION}

Maritime systems, like ships and all their subsystems, are typically operated in a harsh and largely variable environment. At the same time, failures in any of the subsystems or components may have large consequences, e.g. high costs (loss of revenues, high logistics costs due to remote locations) or environmental impacts. The number of failures in this sector of industry is nowadays typically controlled by performing a lot of preventive maintenance. By replacing the components in time, failures can be prevented. However, this is a rather expensive policy when the operational profile is largely varying. The preventive maintenance intervals must be set to very conservative values to assure that also severely loaded subsystems do not fail. This is a costly process, but it also limits the availability of the system, as it must be available for maintenance tasks quite often.

In addition, the associated service logistic process is also quite inefficient. The conservative maintenance intervals require that large numbers of spare parts are held in stock, leading to high inventory costs. But the unexpected failures that do still occur often lead to unavailability of spares, requiring costly emergency shippings or leading to long down times of the system.

To improve this process, reduce the maintenance and logistic costs and at the same time increase the system availability, a better prediction of failures for systems operated under specific conditions is required. Only when a prognostic method is available to provide such a prediction, maintenance can be performed in a just-in-time manner and the associat- ed logistic process can be organized in an optimal manner.

This paper will address the development of a predictive maintenance concept for maritime assets, aiming to be connected to the optimization of the service logistic process (Eruguz et al., 2015), as is shown schematically in Figure 1. A number of models for various types of components will be introduced. However, a focus on developing advanced prognostic models, either data analytics or physicsof-failure based, will not automatically lead to a more effective maintenance policy: it will be shown that more than just models is required to achieve that.

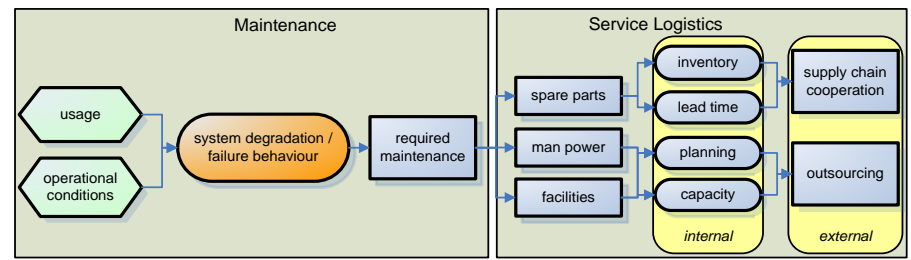

Figure 1. Relation between predictive maintenance and service logistics process.

Therefore, in this paper also other issues related to the development and application of these models will be discussed. Typically, the following issues are encountered in such a development trajectory: (i) critical part selection, (ii) predictive modelling (datadriven or physics based), (iii) monitoring / data collection, (iv) model validation and ( $v$ ) making the business case. After discussing the more generic challenge of the system versus component level in section 2, each of these issues will be treated sepa- 
rately in sections 3 to 7 of this paper. Section 8 will contain a discussion of the results and forwards the conclusions of this work.

\section{SYSTEM VERSUS COMPONENT LEVEL}

Ships and other maritime systems are typically complex systems containing large numbers of subsystems and components. A system diagram of a typical (naval) ship is shown in Figure 2. The ship is subdivided into five main functions (e.g. platform functionality), which are each again subdivided into one up to four subfunctions (e.g. mobility / propulsion). Finally, each of the subfunctions is realized with 1 up to 11 installations, like a diesel engine, sewage system or navigation radar.

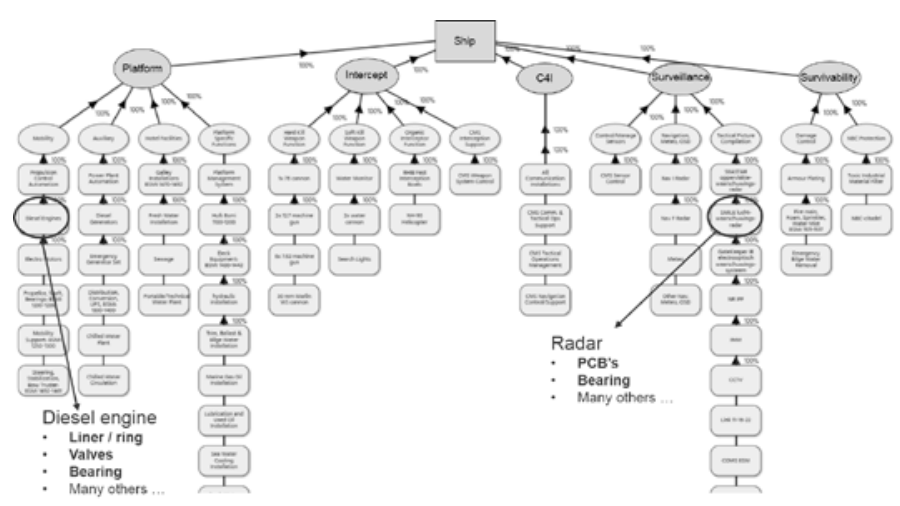

Figure 2. Naval ship system diagram, showing the complexity and indicating some of the analyzed components.

However, it should be realized that this is not the lowest level, as each of the installations consists of numerous components. For the diesel engine, these are e.g. bearings, liners, pistons, etc. Prognostic methods, especially physics of failure based methods, are typically developed at this lowest (component) level. But asset owners and operators are interested in the functioning and maintenance optimization on the highest (system / ship) level.

This immediately demonstrates one of the largest challenges in predictive maintenance: how can the system level maintenance optimization be connected to the component level prognostic methods ? For an effective preventive maintenance concept, ideally prognostic models for all individual components would be available. Firstly, this would enable the prediction of any failure occurring in the ship, giving the operator the opportunity to take appropriate action before the actual failure occurs. Secondly, it would provide the input that mathematical maintenance modelling and clustering methods (e.g. Eruguz et al. (2016)) require to optimize the associated service logistic process, see Figure 1.

However, due to the large numbers of components and the effort required to develop prognostic methods, this full coverage of all components is not fea- sible in the practice of maritime assets. The consequence is two-fold: (i) a suitable selection method is required to select those components for which developing prognostic methods is useful, and (ii) an approach is required to generate the complete input for optimization methods (typically failure rates at different operating conditions). The first issue, i.e. the critical part selection, will be discussed in the next section. The second issue of incomplete input for optimization methods can be solved as follows.

For a limited number of components accurate models or relations for the failure rate at different operating conditions can be derived. This can be physics-of-failure based models or data analytics relations derived from large data sets, as will be discussed in section 4 . For the remaining components (typically the majority, see Figure 1), basically two options remain.

The first option is to assume a failure rate based on information from the OEM of the system. Typically this will be a constant failure rate (e.g. expressed as a mean time between failures - MTBF), as only in exceptional cases the OEM provides a differentiation in failure rates at different conditions.

A second option would be to base these failure rates on expert opinion. Operators of specific systems typically know quite well, based on experience, how the system behaves (and fails) under certain circumstances. By quantifying this experience, preferably obtained from a number of different experts, into a failure rate, the optimization method can be fed.

Both options, however, provide estimates of the failure rates, which in most cases are less accurate than the values obtained from prognostic methods. This means that it is essential to differentiate between critical and non-critical components, as an inaccurate estimate for a critical component could lead to very unrealistic results. This again illustrates the need for a critical part selection method, as will be discussed in the next section.

\section{CRITICAL PART SELECTION}

In the previous sections the need for a critical part selection method was illustrated. Traditionally, failure mode, effect and criticality analyses (FMECA) are executed to assess the criticality of components, expressed in terms of Risk Priority Numbers (RPN). That approach was also initially applied to the maritime systems, like vessel propulsion systems and a radar system, in this project. However, prioritizing subsystems or components for developing prognostic models just on the basis of RPN appeared to give some complications. 


\subsection{Complications}

Firstly, risk is a combination of frequency and impact. A failure with moderate effect that occurs regularly can therefore represent a similar risk as a (potential) failure with large impact, but a low probability of occurrence. For the first type of failure a predictive method would be very useful, while for the second type of failure the low probability of occurrence would probably not justify the development effort of such a method.

Secondly, for a complex system a FMECA analysis can easily become very extensive and time consuming, especially when it is being executed down to the component level. This relates to the discussion in section 2 on the system vs. component level. Higher level approaches, like quick RCM or streamlined RCM may solve the time issue, by only focusing on subsystem or assembly level. But at the same time they do not guarantee that all critical components have been identified.

Thirdly, in a FMECA the quantification of the RPNs is based on a predefined scaling of occurrence and severity. However, the severity or impact can be quantified in many different ways, e.g. in terms of down-time, costs, environmental effects or safety. Depending on the application, one of these factors might be dominant, but in many cases a weighted combination of these factors will quantify the impact. In the maritime applications studied in this project, different situations have been encountered.

Finally, it was discovered in the present project that only being a critical component still does not make a component a suitable candidate for predictive maintenance. A prognostic method is only useful when the prediction of failures actually enables to extend or reduce the standard maintenance intervals. In case of an extension, costs are reduced, while a reduction typically means that a failure that otherwise would have occurred can be prevented, thus leading to an increase in system availability.

However, in maritime systems, as is also the case in some other sectors of industry, maintenance tasks are commonly clustered in time. This means that at a predefined interval, a range of components is replaced. The advantage is that the engineer only has to travel to the ship once, which means that the socalled set-up costs can be shared by all components. But the drawback of this practice is that extension of the maintenance interval of one (critical) component based on a prognostic method means that it will move outside the cluster of activities, unless the interval can be extended with the same amount for all components in the cluster.

\subsection{Solutions to the complications}

To tackle the first problem, Lee et al. (2014) proposed the 4-quadrant method, in which failures can be plotted along two axes: the failure frequency and the failure consequence (which is expressed in terms of e.g. costs or down-time). Tiddens et al. (2017b) proposed a modification of this method, using different boundaries between the four regions. Such a graph is shown for a series of failures of a vessel propulsion system in Figure 3. The failures with low impact can be addressed by either applying regular OEM prescribed maintenance (for low frequency failures) or arranging a sufficient amount of spare parts (for high frequency failures). Unacceptable failures are those failures that either combine high failure frequencies with high impact, or only have very high impact or very high frequencies. Failures in that region of the graph should be prevented by all means, so a redesign or modification of the system is the best option. Finally, the failures that remain in the fourth quadrant, representing moderate to high impact at moderate to high frequencies, are (in principle) suitable candidates for predictive maintenance. However, in the FMECA analyses performed on the propulsion system, many potential failures with high impact were mentioned, but these did not occur yet in the vessels in operation for the past 5-10 years. Examples are gear box failures and propeller shaft fracture. Developing prognostic methods for these type of failures will therefore probably never pay back. It is therefore proposed to exclude the low frequency failures from the $4^{\text {th }}$ quadrant, and define the region with potential for predictive maintenance to be the (red) dashed region in Figure 3.

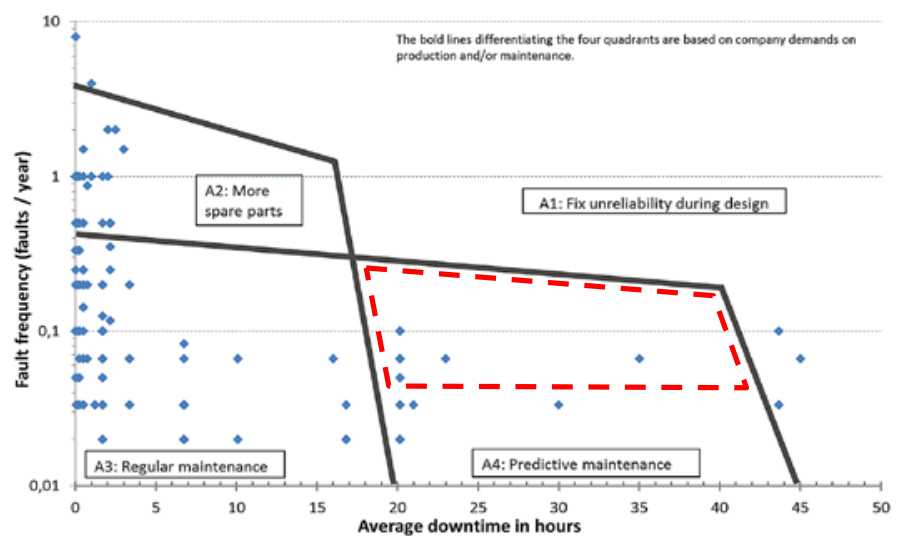

Figure 3. Modified 4-quadrant method, showing a series of failures for a vessel propulsion system. The dashed region indicates the failures that are suitable for predictive maintenance.

The second problem of reaching a sufficient level of detail while at the same time not spending too much time on the FMECA analysis could not be solved completely in this project. Four FMECA sessions with different asset owners have been completed, each lasting for one half up to one day. To reduce the effort, not the complete ship was taken as starting point, but a subsystem. More specifically, the propulsion system (engine, generator, shaft, propeller) and a radar system have been analyzed, while the facilitator ensured that sufficient focus was kept during the analysis. However, the quality of the analysis in that case depends on the facilitator competence, the 
method does not guarantee an appropriate result. The authors therefore presently work on a recursive FMEA method that enforces to focus on the critical components without the burden of doing a detailed analysis on the complete system.

The third problem was the factor to be used to quantify the impact of a failure. For both naval applications and piloting services, availability of the ships is by far the most important, while costs only play a secondary role. For these type of ships and systems, the impact of failures should therefore be expressed in terms of (system) down-time. Activities like harbor towing or hydrographic surveying services are more commercial, so costs are much more important for the ships used there. However, compared to the huge costs due to production loss (unable to deliver service for a certain amount of time), the direct costs of a (sub)system failure (labor and spare parts costs) are almost negligible. Therefore, also for these ships, the down-time is the most important factor determining the impact of a failure. But for other applications, e.g. aircraft or nuclear plants, a combination of factors might be decisive for the impact. In that case, more advanced methods, like multiple criteria analysis, might be required to determine the most critical components.

The final problem, the clustering of activities, was encountered in this project for some diesel engine components. The cylinder liner was identified to be a critical component (i.e. in the $4^{\text {th }}$ quadrant of Figure 3 ), and therefore seemed to be a suitable component for a prognostic method. However, in practice, when the liner is replaced, also the piston rings and exhaust valves are replaced. Moreover, in this case the exhaust valves appeared to be the most critical components, so the interval length of this cluster was governed by the valve service life. Therefore, if a prognostic method for the liner would advise to extend the interval, the engine still has to be overhauled to replace the rings and valves. That would minimize the benefit of such a method, which makes the liner a much less suitable candidate for predictive maintenance. In this case, the exhaust valves would be a better candidate, as these components govern the interval length. Extension of the valve interval, and the whole cluster with it, would then directly give a cost benefit.

To conclude this section, it appears that the critical part selection is not a trivial process. Based on the experience in this project with maritime systems, the following procedure is proposed:

1. Perform a FMECA on the system with sufficient level of detail (down to component level), and determine RPN for each failure;

2. Plot the failures with the highest RPN in the 4 quadrant graph (Figure 3) and use the most appropriate quantity on the impact axis;
3. Determine the boundaries of the quadrants and the dashed region based on the application specifics;

4. Check for each of the potential candidates (from the dashed region in 3) whether they are

a. independent of any cluster of activities, or

b. governing the length of the cluster interval

\section{PREDICTIVE MODELS}

Once the critical parts in a system have been selected, prognostic models for these components can be developed. While nowadays many models are datadriven, based only on mathematical relations between collected parameters (Lebold and Thurston, 2001), most of the models presented in this work are based on the physical degradation or failure mechanisms, like wear, corrosion and fatigue (Tinga, 2010). The author previously presented similar models in other application fields (Tinga, 2013). In this section, models for diesel engine parts like the cylinder liner and cylinder rings will be presented, as well as electrical parts of radar systems, i.e. printed circuit boards.

\subsection{Diesel engine cylinder liners}

In a diesel engine, the liner covers the inside of the cylinder, in which the piston is reciprocating, see Figure 4. The piston contains a number of rings, which are in lubricated contact with the liner. The reciprocating motion maintains the lubricant oil film, but at the top and bottom reversal points, the film thickness is less, and mechanical wear can occur due to the relative motion of the two metal parts.

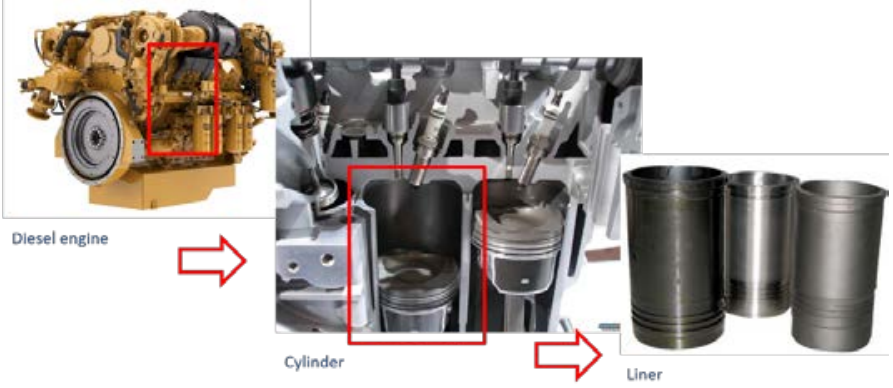

Figure 4. Position of cylinder liner and piston rings in a diesel engine.

A model has been developed (Amoiralis, 2015) that describes the physical degradation processes of sliding wear occurring at the interface between liner and ring. The model applied is the Archard wear model (Archard, 1953), relating the wear volume (V) to the normal force $(F)$, sliding distance $(s)$ and wear parameter $(k)$ :

$V=k F s$ 
This model requires a number of inputs, that can be related to either the properties or the operating conditions of the specific engine:

- The normal force $F$ is directly related to the pressure in the cylinder, and therefore depends on the engine operating condition (e.g. power).

- The sliding distance s can be related to the dimensions of the engine components. The sliding distance per cycle that the liner experiences is equal to two times the accumulated width of the cylinder rings, that pass a certain location on the liner two times each cycle (up and down). Further, the speed of the engine (rpm) determines the number of passings per time unit.

- The wear parameter $k$ is the proportionality constant, which depends on a number of factors. It can be estimated for the specific combination of materials (liner and rings) and wear mechanism. If for example the lubricant is contaminated with particles, the mechanism will switch from adhesive to abrasive wear, which will change the value of the wear parameter.

- Finally, in a lubricated contact the amount of wear will be negligible, since there is no metal-to-metal contact. This means that only wear occurs when the lubricant film thickness of the cylinder is lower than the critical film thickness. In case of adhesive wear, the critical film thickness equals the liner surface roughness, while for abrasive wear it is equal to the particle size. The calculation of the wear depth thus also requires the determination of the film thickness distribution along the liner surface, which depends on piston tilt and speed, temperature, surface roughness and oil viscosity.

The relation between these parameters is visualized in Figure 5.

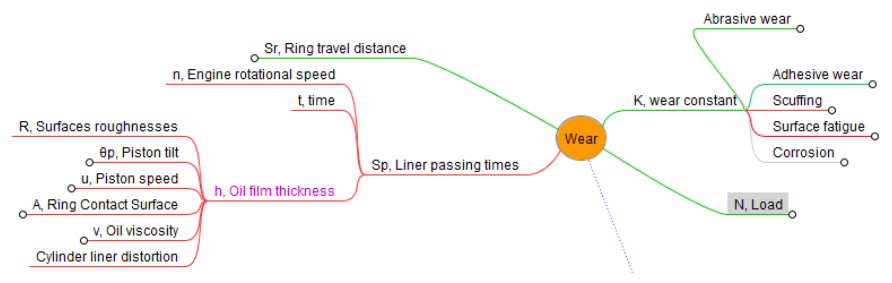

Figure 5. Overview of factors in wear model for cylinder liner.

Application of the model to a specific engine now firstly requires to specify the engine characteristics (e.g. dimensions, material) to determine some model constants. After that, the engine operating conditions, either constant in time, or specified by a certain operating profile, can be used as input for the model.

The first step is then to calculate the oil film thickness along the liner surface. This is shown in Figure 6 for a certain engine and operating condition, in this case as a function of crack angle (a four stroke diesel engine rotates over 720 degrees each cycle, so crank angles of 90, 270, 450 and 630 degrees represent the same location on the liner). Also the critical film thicknesses for adhesive and abrasive wear are indicated. Note that the absolute values of the film thickness depend on several model parameters, which have to be determined from experiments. In this stage of the modeling process, typical values are used.

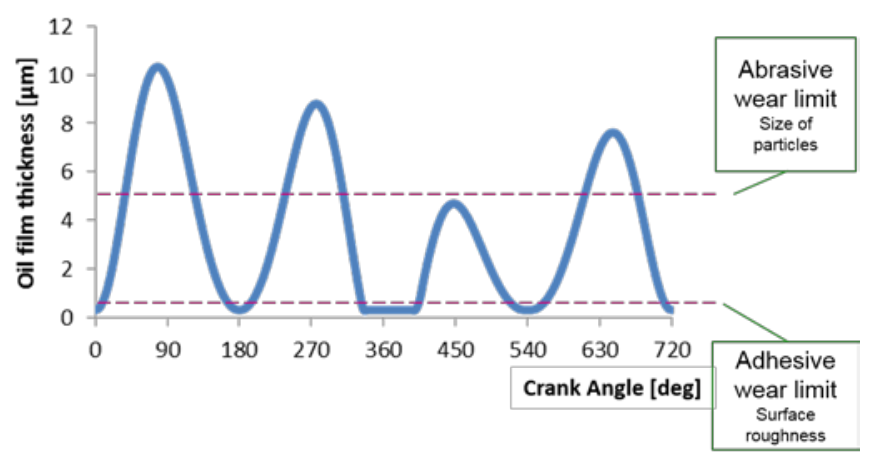

Figure 6. Film thickness variation with crank angle, including wear limits for adhesive and abrasive wear.

This graph clearly shows that without oil contamination, wear will only occur in some small regions around the reversal points of the piston $(180,360, .$.$) .$

Using this variation of oil film thickness, and together with the calculated normal force (as obtained from the cylinder pressure), the wear rate distribution along the liner can be calculated for three different artificial operating scenarios, see Figure 7a.
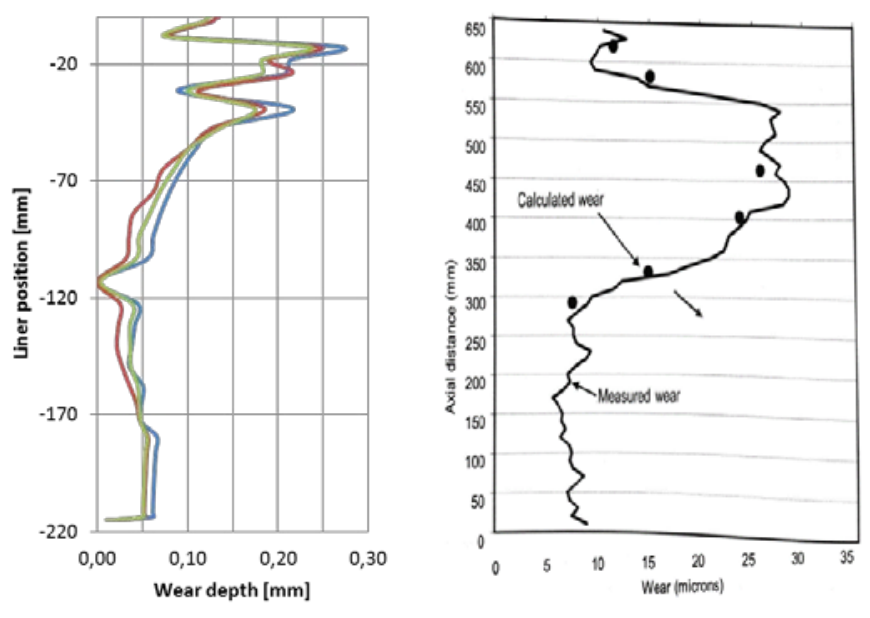

-Schen A - Schen B — Schen C

Figure 7. a) Calculated wear depth along the liner position for three different operating scenarios, b) measured wear profile for a similar engine (Lakshminarayanan and Nayak, 2011).

These scenarios all represent 15000 hours of operation, but differ in their division over high / medium / low speed, respectively high / medium / low load, see Table 1. For comparison, a measured wear profile from a real diesel engine is shown in Figure 7b, showing a very similar variation with liner position. Table 1 also shows the calculated remaining service life of the liners, assuming a replacement at $0.3 \mathrm{~mm}$ wear depth. 
The final step in the predictive method is to relate the wear of the liner to variations in operating conditions of real engines. This has been done for a ship with three diesel engines (SB - starboard, CE - center and PS - portside) operating in four different profiles. The distribution of operating hours over these four profiles is shown in Figure 8a, which is based on the monitoring data obtained from the asset owner.

Table 1. Calculated wear depth and remaining life after 15000 hrs for three different operating scenarios.

\begin{tabular}{lcc}
\hline Scenario & $\begin{array}{c}\text { Max. wear } \\
\text { depth (mm) }\end{array}$ & $\begin{array}{c}\text { Remaining } \\
\text { life (hrs) }\end{array}$ \\
\hline A (high speed / high load) & 0.271 & 1603 \\
B (high speed / var. load) & 0.247 & 3224 \\
C (var. speed / var. load) & 0.236 & 4103 \\
\hline
\end{tabular}

During the simulated period the SB engine has been replaced by a new one, explaining the double bar for SB in the diagram. Figure $8 \mathrm{~b}$ shows the calculated evolution of the wear depth for the three engines over time. The plot clearly shows variations in wear rate, which can be associated to changes in operating profile. Further, it can be concluded that the three engines are operated differently, and thus also show a considerable difference in wear behavior.
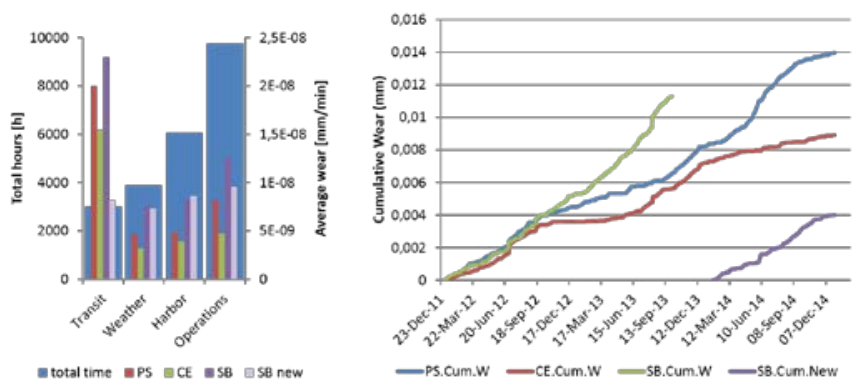

Figure 8. a) Distribution of operating hours over three engines and four operating profiles, b) calculated wear depth evolution over time for the three engines.

Once validated (see section 6), these kind of calculations can serve as prognostic methods for the liners, as monitoring the operational profile of the engines enables to closely follow the wear evolution and assess the remaining service life. That information ultimately will make just-in-time maintenance feasible.

\subsection{Radar system printed circuit boards}

Contrary to a diesel engine, which consists of mainly mechanical components, a radar system contains mainly electrical components. The phased array radar system on a navy ship considered here contains a large number of so-called column assemblies (CA), which in their turn contain a number of printed circuit boards (PCB), see Figure 9. As the PCBs of the
CA appear to fail regularly and unexpectedly, a prognostic method for these components could increase the radar availability and assist in improving the logistic process of these parts. Again a physicsof-failure based approach will be followed here.

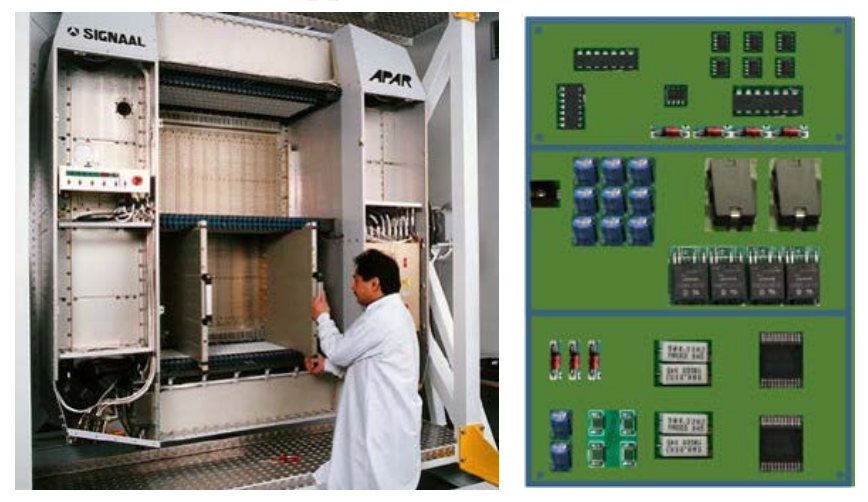

Figure 9. a) Radar system during removal of one of the column assemblies (CA), b) Typical layout of printed circuit board.

The development (Politis, 2015) started with a root cause analysis to determine the actual failure mechanisms responsible for the functional failure of the PCB. These appeared to be thermal fatigue, driven by changes in operating temperature of the PCBs and mechanical fatigue due to vibrations. For both mechanisms, models are available in literature.

The thermal fatigue is modelled with the MansonCoffin relation, adapted for crack growth in solder materials (NIST/SEMATECH, 2012). The number of (temperature) cycles to failure $N$ is a function of the magnitude of the temperature cycle $(\Delta T)$, the maximum temperature $\left(T_{\max }\right)$ and the cycling frequency $(f)$ :

$$
N=A f^{-a} \Delta T^{-b} G\left(T_{\max }\right)
$$

The constants $A, a$ and $b$ are model parameters. The mechanical fatigue due to vibrations is modelled according to (Steinberg, 2000):

$$
N_{0}=N_{c}\left(\frac{Z_{3 \text { dimit }}}{Z_{0}}\right)^{6.4}
$$

The number of cycles to failure $N_{0}$ at a certain vibration induced peak amplitude displacement $Z_{0}$ is a certain fraction of a reference number of cycles $N_{c}$ at a critical displacement amplitude $Z_{3 \text { olimit. }}$ The latter value can be determined from the dimensions of the PCB and the type and position of the component considered. The actual displacement is obtained from the natural frequency of the board and the power spectral density (PSD) of the vibration encountered by the PCB.

Now the models have been established and the model constants have been set (using the actual dimensions and characteristics of the PCB and suggested values from literature), the loads on the $\mathrm{PCB}$ have to be determined. As the PCB is in the radar 
during operation of the ship, the variations in both temperature cycles and vibration levels have to be determined. For that purpose, tests have been performed with the actual PCBs. By simulating the operational cycles in a testing machine, a thermal camera could be used to measure the temperature cycles (see Figure 10a). As the number of operating cycles is limited, the input parameters for the model $(\Delta T$, $T_{\max }, f$ ) can now directly be linked to the on/off switching of the radar and the specific operational mode. In addition, an accelerometer has been placed inside the radar (see Figure 10b), and vibration measurements have been performed for a range of operating conditions (ten Zeldam, 2016): various sea states, with diesel engine or gas turbine propulsion.
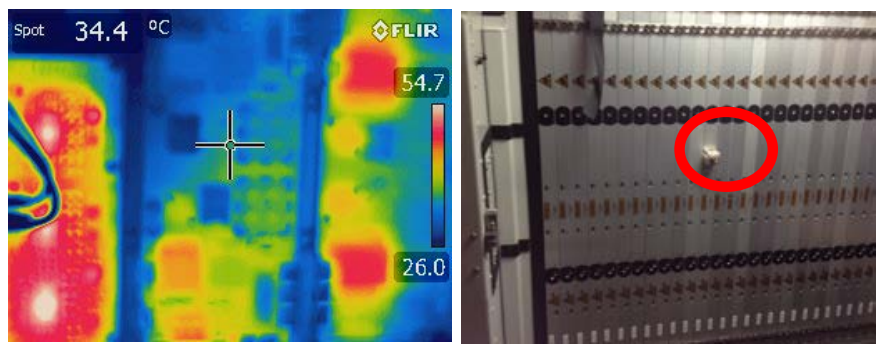

Figure 10. a) Thermal camera image of temperature distribution over PCB components, b) accelerometer placed in the radar.

As the switching history and vibration levels are not continuously monitored (see also section 5), a limited number of operating profiles are defined. For each profile, using expert opinion, the switching frequency and vibration level variation (based on the different measured operational conditions) are specified. It is then possible to compare different scenarios, each containing a certain sequence of operating profiles with specified duration. The damage accumulation can be calculated with the models in equations (2) and (3), and the remaining useful life of the PCBs can be estimated. To take into account the uncertainty in model parameters and usage, a Monte Carlo simulation is performed to quantify the uncertainty in the calculated life time.

The results for four different operating profiles is given in Table 2, comparing diesel and gas turbine propulsion, as well as various speeds and weather conditions. It is clear that gas turbine propulsion is less damaging for the radar PCBs, as the vibration levels in the ship are lower in that case.

Table 2. Calculated PCB damage (per time unit).

\begin{tabular}{lc}
\hline Scenario & Damage \\
\hline A (Die / medium speed / medium weather) & 1.41 \\
B (Die / medium speed / bad weather) & 5.55 \\
C (GT / medium speed / medium weather) & 0.70 \\
D (GT / high speed / medium weather) & 2.10 \\
\hline
\end{tabular}

Die = diesel, $\mathrm{GT}$ = gas turbine propulsion

\section{MONITORING AND DATA COLLECTION}

Once prognostic models are available, application in practice requires the monitoring of appropriate parameters on usage and loading of the systems. The type of data required depends on the model. For the liner model discussed in section 4.1, the variations in engine operating conditions must be monitored, i.e. engine speed and load as a function of time. For the PCB model in 4.2, the vibration levels and of on/off switching history are required.

Although both requirements in principle can be met by deploying rather simple sensors or data acquisition systems, in maritime systems this appears not to be common practice. Typically, three types of challenges are encountered. Firstly, many systems nowadays contain built-in monitoring systems, but the data is often not stored over time. This means that a history of some days up to two months is available, typically for diagnostic purposes in case of failures, but the data is overwritten when it has reached the expiry date. This means that the complete time history of the usage is often not available.

Secondly, the sampling frequency is often inappropriate. For a quickly varying diesel engine parameter like speed or load, one measurement every hour is useless. Thirdly, the quality of the data is often inadequate, especially when data has to be entered manually by an operator of the system. For example, registrations of failures are often not entered directly into the maintenance management system, which often leads to wrong dates / time in the registrations. Also the cause and details of the failure are often incompletely registered, which makes additional analyses quite challenging.

For the two cases discussed in section 4, the required data was available (engine) or could be made available with limited effort (radar vibrations), but these cases seem to be exceptions: for most systems on board of ships, detailed data on usage and loads is not readily available. The concept of defining a number of functional usage profiles and register the relative occurrence of those partly solves the lack of continuous monitoring data.

To conclude, any company that has the ambition to shift to a more predictive form of maintenance will have to invest in a proper measurement and registration of the variations in operational profile.

\section{MODEL VALIDATION}

One of the biggest challenges in applying predictive models is the validation of the method. To check the accuracy, model predictions will have to be compared with real failures. However, this is only feasible when (i) the complete usage history of the system or component is available, and (ii) the service life or 
(preferably) the decrease in condition of the component can be assessed on a regular basis.

The first requirement, that was also discussed in the previous section, is a problem for the PCB model (section 4.2) validation. Although quite a number of failures have been registered in a certain operating period, the exact operating history of each individual PCB is not available. This is one the one hand caused by the fact that the internal radar monitoring system only stores the data for a limited period of time. On the other hand, PCBs are exchanged between radars on different ships, which further complicates the reconstruction of the load history. For that reason, complete validation of the radar PCB model has until now not been successful.

The second requirement is a problem with the cylinder liners. These liners are replaced at predefined maintenance intervals, but their actual condition at replacement is not assessed. This means that, although in some cases the load history is known, the condition or remaining life time is not available. To solve this problem, a measurement campaign is now being prepared, aiming to assess the wear profile of the liners that are replaced. The measured wear depth, and its variation along the liner, can then be compared to the model prediction, which will enable the validation of the method.

\section{BUSINESS CASE}

The final challenge in predictive maintenance is to make the business case. The potential of predictive maintenance is clear, but it is still very difficult for a specific case to quantify whether the expected benefits will exceed the required investments. In this project, a method has been developed to do that analysis. This method will be presented in a separate paper (Tiddens et al., 2017a).

\section{CONCLUSIONS}

Application of predictive maintenance to maritime systems has a clear potential in increasing availability and reducing maintenance and logistic costs. However, in addition to the challenge of developing appropriate models for the critical components, also a number of additional challenges must be tackled. This paper has discussed the issues of critical part selection, monitoring and data collection and model validation, and has indicated the importance of making a business case. Before predictive maintenance can be applied in a broad sense in this sector, these issues should be solved first.

\section{ACKNOWLEDGEMENTS}

The work presented in this paper has been executed in the project "Advanced Maintenance and Service Logistics for Maritime Assets” (MaSeLMA) funded by the Dutch Institute for Advanced Logistics. The cooperation with Thales, Fugro, Loodswezen, the RNL Navy and Pon Power is greatly acknowledged.

\section{REFERENCES}

Amoiralis, F. 2015. Development of predictive maintenance concepts for maritime assets. Enschede: University of Twente (unpublished work).

Archard, J. F. 1953. Contact and rubbing of flat surfaces. Journal of Applied Physics, 24, 981-988.

Eruguz, A. S., Tan, T. \& Van Houtum, G. J. J. a. N. 2015. A survey of maintenance and service logistics management : classification and research agenda from a maritime sector perspective. BETA working paper 489. Eindhoven: Eindhoven University of Technology.

Eruguz, A. S., Tan, T. \& Van Houtum, G. J. J. a. N. 2016. Integrated maintenance and spare part optimization for moving assets BETA working paper 500. Eindhoven: Eindhoven University of Technology.

Lakshminarayanan, P. A. \& Nayak, N. S. 2011. Critical Component Wear in Heavy Duty Engines, Singapore, Wiley.

Lebold, M. \& Thurston, M. 2001. Open standards for condition-based maintenance and prognostic systems. 5th annual Maintenance and Reliability Conference.

Lee, J., Wu, F., Zhao, W., Ghaffari, M., Liao, L. \& Siegel, D. 2014. Prognostics and health management design for rotary machinery systems - Reviews, methodology and applications. Mechanical Systems and Signal Processing, 42, 314-334.

Nist/Sematech. 2012. e-Handbook of Statistical Methods: http://www.itl.nist.gov/div898/handbook/index.htm [Accessed March 2015.

Politis, M. 2015. A prognostic method for electronic components of radar systems. PDEng thesis, Eindhoven University of Technology.

Steinberg, D. 2000. Vibration analysis for electronic equipment, John Wiley \& Sons.

Ten Zeldam, S. G. 2016. Predicting the (remaining) lifespan of electronical components of radar systems. BSc thesis, Netherlands Defence Academy.

Tiddens, W. W., Braaksma, A. J. J., Brouwer, O. \& Tinga, T. 2017a. The business case for condition-based maintenance: exploitation vs exploration. ESREL 2017 - European Safety and Reliability Conference Portoroz, Slovenia: ESREL.

Tiddens, W. W., Braaksma, A. J. J. \& Tinga, T. 2017 b. Towards Informed Maintenance Decision Making: Guiding the Application of Advanced Maintenance Analyses. Optimum Decision Making in Asset Management. Hershey, USA: IGI Global.

Tinga, T. 2010. Application of physical failure models to enable usage and load based maintenance. Reliability Engineering and System Safety, 95, 1061-1075.

Tinga, T. 2013. Predictive maintenance of military systems based on physical failure models. Chemical Engineering Transactions, 33, 295-300. 